\title{
Ecrire des images
}

Writing images

\section{François Aubart}

\section{(2) OpenEdition}

\section{Journals}

Édition électronique

URL : http://journals.openedition.org/imagesrevues/4220

DOI : 10.4000/imagesrevues.4220

ISSN : 1778-3801

\section{Éditeur :}

Centre d'Histoire et Théorie des Arts, Groupe d'Anthropologie Historique de l'Occident Médiéval, Laboratoire d'Anthropologie Sociale, UMR 8210 Anthropologie et Histoire des Mondes Antiques

\section{Référence électronique}

François Aubart, « Ecrire des images », Images Re-vues [En ligne], Hors-série 6 | 2018, mis en ligne le 08 avril 2021, consulté le 08 avril 2021. URL : http://journals.openedition.org/imagesrevues/4220 ; DOI : https://doi.org/10.4000/imagesrevues.4220

Ce document a été généré automatiquement le 8 avril 2021.

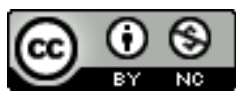

Images Re-vues est mise à disposition selon les termes de la Licence Creative Commons Attribution Pas d'Utilisation Commerciale 4.0 International. 


\section{Ecrire des images}

Writing images

François Aubart

Lire et imaginer 
1 Aux États-Unis, à la fin des années 1970, commencent à apparaître les travaux d'une génération d'artistes nés entre 1945 et 1955. Leurs œuvres copient, imitent ou s'inspirent des images de la publicité, de la télévision et du cinéma. Parce qu'ils utilisent des représentations préexistantes, puisées dans leur environnement culturel, on utilise parfois l'appellation d'appropriationistes pour nommer ces artistes. Ce terme désignerait une pratique basée sur la copie de productions d'autres artistes, prolongeant ainsi celle de leurs aînés tels que Elaine Sturtevant ou Richard Pettibone. Pourtant ce terme semble inapproprié puisque, bien souvent, les œuvres en question ne sont pas des copies d'une image précise

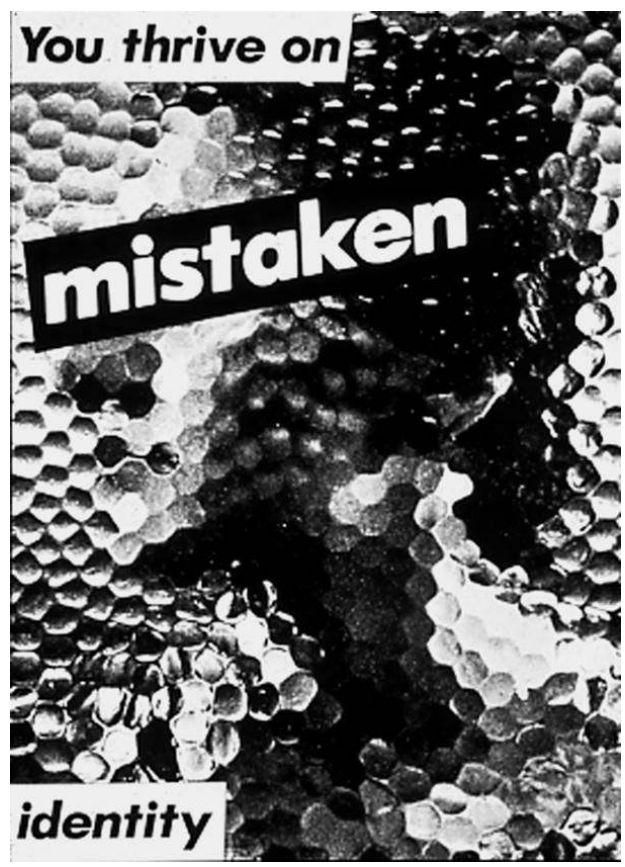
mais tendent plutôt à reproduire une typologie générique. Par exemple, il est impossible de dire précisément qui est plagié dans les autoportraits de Cindy Sherman. Les images que Richard Prince ou Barbara Kruger empruntent dans des magazines ou des livres n'ont, sauf cas exceptionnels, pas d'auteurs revendiqués.

2 L'exposition The Pictures Generation, 1974-1984 organisée en 2009 au Metropolitan Museum a entériné un autre terme souvent utilisé avant cela pour nommer ces artistes. Celui-ci convoque le titre de l'exposition Pictures organisée en 1977 par Douglas Crimp qui non seulement est une des premières occasions de réunir certains de ces artistes et qui en plus formule, dans un texte maintes fois republié, les premiers enjeux théoriques qui les accompagnent ${ }^{1}$. En fait, le terme Pictures Generation a l'avantage de signaler précisément ce qui est en jeu dans ces travaux : des images.

3 Celles qu'utilisent ces artistes sont caractérisées par un mode de production qui ne relève pas de l'enregistrement direct de la réalité. Les images de la publicité et du cinéma convoquées dans leurs œuvres ne sont que très rarement documentaires. Ce sont le plus souvent des représentations mises en scène et composées pour représenter un monde imaginaire ${ }^{2}$.

4 Ici nous aimerions proposer une hypothèse. L'intérêt que portent les artistes de la Pictures Generation pour ces images mensongères, factices et idéales s'accompagne d'une attention à ce qu'elles font à leurs publics. Elles suscitent du désir et de la fascination. Qui plus est, il nous semble que cet intérêt est manifeste dans les productions textuelles des artistes de la Pictures Generation. Bien que l'écriture ne soit pas un domaine qui relève de la production d'images factuelles, elle fait apparaître mentalement. Or, beaucoup d'artistes de cette génération produisent des textes qui accompagnent les recherches plastiques qu'ils mènent sous la forme de photographies, d'installations, de films. Selon des modalités différentes, les textes de Jack Goldstein, Jenny Holzer, Barbara Kruger, Louise Lawler, Sherrie Levine, Matt Mullican ou Richard Prince tentent d'affecter leurs lecteurs, attisent leur imaginaire et leurs émotions. Autrement dit, nous souhaitons montrer que lorsque les œuvres des artistes de la 
Pictures Generation ne sont pas visuelles elles ne signalent pas moins ce que font les images convoquées dans leurs travaux.

5 À titre d'exemple, il semble assez révélateur que Barbara Kruger, avant de réaliser les affiches faites de slogans et d'images qui la rendront célèbre, publie en 1978, à compte d'auteur, un livre qui présente des textes en vis-à-vis de photographies de bâtiments d'habitation's.

Fig. 1

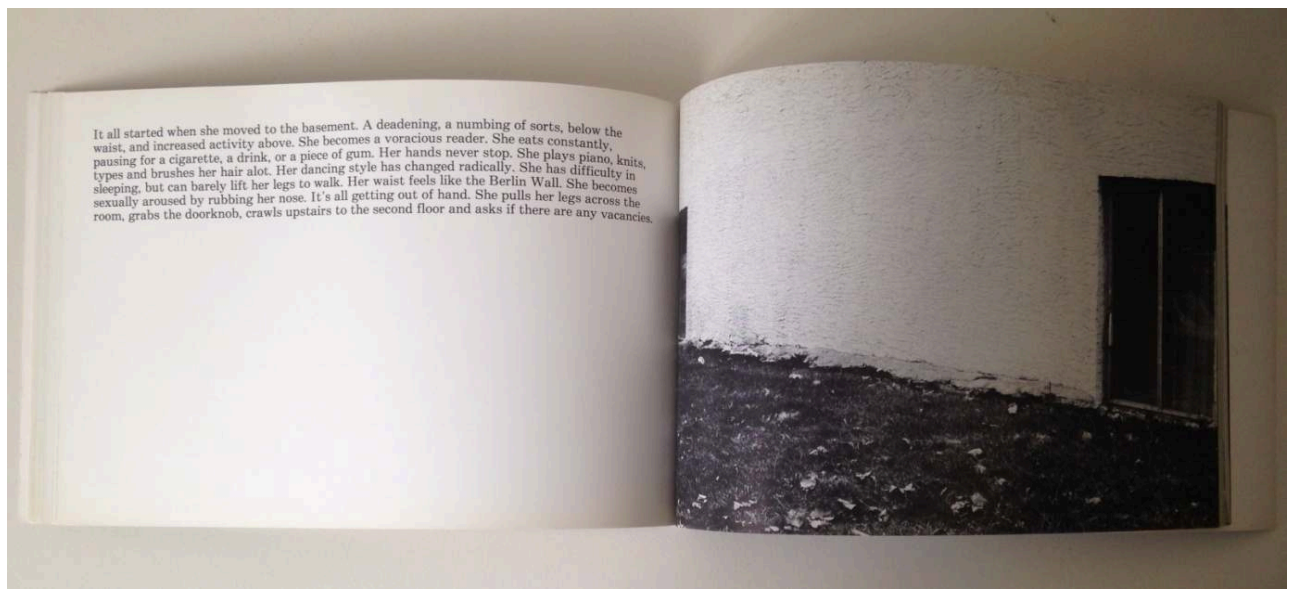

Barbara Kruger, Pictures/Reading, 1978

$6 \quad$ Picture/Readings (Fig.1), présente un court texte sur chaque page de gauche et, sur celles de droite, une photographie de façade d'immeuble cadrée très serré, comme un gros plan ou un agrandissement. On lit ainsi de brèves histoires qui racontent la vie, les sentiments et les attitudes de personnages décrits dans des scènes et qui pourraient se dérouler dans les bâtiments représentés en vis-à-vis.

7 On lit par exemple à propos de Hal, l'un des personnages, qu' «Il pense à la journée d'hier au travail. Il est assistant à la banque. Son patron lui criait dessus devant les guichetiers hier. Il s'est senti incompétent et désespéré» ${ }^{4}$. L'expression des états d'âme de fin de journée de Hal est accompagnée de la photographie d'une façade de bâtiment. Dans aucune des deux pages nous ne voyons Hal lui-même ni son environnement. Mais nous pouvons nous en faire une représentation parce que la scène est assez précise pour paraître crédible et en même temps assez générique pour être imaginée. Autrement dit, dans Picture/Readings les textes et les images ne présentent pas des faits avérés et vérifiables. Le but de leur association est de faire imaginer aux lecteurs une situation plausible et attachante pour lui communiquer des sentiments.

\section{Déclarations par imitation}

8 Au début des années 1980, Sherrie Levine écrit des Statements [Déclarations] qui sont publiées dans des magazines ou des catalogues ${ }^{5}$. Ces textes de quelques phrases signées de l'artiste ressemblent à des argumentaires expliquant son travail. C'est alors une pratique courante, exploitée notamment par certains artistes conceptuels. Leurs œuvres sont souvent le résultat d'un protocole ou d'un processus logique qui nécessite d'être présenté. Le critique d'art Benjamin Buchloh voit dans cette utilisation d'un langage strictement informatif une tentative d'enrayer l'expérience esthétique par 
"l'introduction d'un langage légaliste et d'un style administratif " ${ }^{6}$. En effet certains de ces artistes exploitent le caractère officiel du style et des documents administratifs et beaucoup écrivent des textes qui présentent leur démarche et leurs intentions pour donner un sens et une cohérence à leurs projets. D'ailleurs ce type de déclarations se développe en même temps que des documents, signés par les artistes, permettant d'attester de l'authenticité des œuvres. Le plus emblématique est celui inventé en 1971 par le galeriste Seth Siegelaub "The Artist's Reserved Rights Transfer And Sale Agreement " [Contrat sur la préservation des droits de l'artiste sur la vente et le transfert]. Conçu comme un moyen de défendre l'artiste, ce document se base sur la loi et sur le droit d'auteur pour valider l'originalité d'une œuvre.

9 À l'inverse, les Statements de Levine n'ont rien ni d'administratif ni de légal. Les mots qui les composent sont empruntés. Ce sont des copies ou des adaptations de textes préexistants. L'un d'entre eux reprend le passage d'une nouvelle d'Alberto Moravia dans lequel la narratrice raconte avoir vu ses parents pendant un rapport sexuel lorsqu'elle était enfant. Horrifiée, sa réaction est de diviser sa personnalité en deux, l'une continue d'être présente au monde tandis que l'autre prend de la distance et l'observe de loin.

10 Sherrie Levine n'est pas l'auteure de ce texte. Néanmoins, lorsqu'elle le convoque, il explique sa démarche qui consiste à copier des productions d'autres artistes. C'est par exemple le cas pour sa série After Walker Evans. Il s'agit de photographies de reproductions d'œuvres de Walker Evans publiées dans un catalogue. Levine en fait l'expérience et en montre des reproductions légèrement dégradées. Ses tirages présentent un léger flou et une qualité qui aurait été honnie par Evans ${ }^{7}$. Autrement dit, Levine propose de regarder les clichés de Evans à distance, comme le fait le personnage de la nouvelle de Moravia. Elle souligne ainsi que les images que fait Walker Evans des paysans appauvris par la crise de la Grande Dépression peuvent devenir des représentations génériques. En montrant ces images, en les redonnant à voir, Levine ne montre pas des sujets singuliers - un homme, une femme ou un enfant - comme le faisait Evans en allant sur place mais l'image générique qui en est faite : l'homme, la femme, l'enfant ${ }^{8}$. Montrées par Levine ces images qui étaient originellement documentaires deviennent des images mises en scène et artificielles. Regardées à distance elles rendent moins compte d'une réalité objective que de la construction sociale d'une représentation générique.

11 Ainsi le Statement de Levine rend bien compte de son travail. Mais, exprimé par métaphore et produit par la copie, il ne remplit pas de fonction légale et ne procède pas de façon administrative. Cette déclaration inauthentique exprime néanmoins une relation au monde qui passe par l'expérience de représentations et de sentiments déjà produits par la photographie ou le roman.

12 Au cours des années 1970-1980 se développent des théories féministes affirmant que dans une société patriarcale le pouvoir est masculin et que le discours, outil de ce pouvoir, appartient aux hommes. Elles s'appuient sur une relecture des théories psychanalytiques de Sigmund Freud et de Jacques Lacan. Le premier a défini l'homme comme le sujet duquel la femme se distingue. Cela se formalise par une absence: le pénis. Cette différence est reconsidérée par Lacan, lequel privilégie le terme de phallus qui implique la notion de représentation du sexe masculin. Pour le psychanalyste structuraliste il s'agit moins de désigner un organe qu'un signifiant qu'on a ou pas. Sa possession ou son manque sont de l'ordre symbolique, c'est le pouvoir de signifier. 
Ainsi la différence sexuelle est envisagée non pas en termes biologiques mais en termes symboliques. Les places assignées par l'ordre social découlent ainsi du langage qui définit et construit la société. Le langage, pouvoir de signification est masculin. C'est à partir de ce constat que se structurent les pensées d'auteures comme Luce Irigaray ou Joan Riviere par exemple. Dans le champ de l'art ces lectures alimentent une interprétation de la représentation et du discours comme des instances de symbolisation, les outils d'un pouvoir patriarcal ${ }^{9}$. La femme, dépourvue de phallus, ne peut être que représentée puisqu'elle est dépourvue de moyens d'expression. Les travaux des jeunes artistes femmes qui à cette période reprennent et manipulent des représentations déjà existantes sont envisagés par certains critiques tels que Craig Owens comme un détournement de ce pouvoir de modeler une représentation de l'autre ${ }^{10}$. C'est ainsi que les travaux de Laurie Anderson, Martha Rosler, Barbara Birnbaum ou Sherrie Levine par exemple sont envisagés comme relevant d'une approche selon laquelle en tant que femmes, dépourvues d'une voix qu'elles puissent revendiquer comme la leur, elles parlent au travers de celle qui ne leur appartient pas. L'imitation est ainsi considérée comme une stratégie féministe que Barbara Kruger exprime en ces termes :

Nous flânons hors du commerce et de la parole et nous sommes obligées de voler le langage. Nous sommes de très bonnes imitatrices. Nous reproduisons certains mots et images et les regardons s'écarter ou coïncider avec vos notions de fait et de fiction. ${ }^{11}$

13 L'artiste exprime là clairement l'idée que les femmes sont reléguées à une place subalterne, dépourvues de moyens d'expression personnels et qu'elles s'en créent un en exploitant une langue qui n'est pas la leur.

En 1981, Levine rédige un Statement composé de phrases empruntées à un texte du peintre Franz Marc et à un passage de «La mort de l'auteur » de Roland Barthes dans lequel le mot «texte» est remplacé par "peinture » et «lecteur» par « regardeur " ${ }^{12}$. Les propos de Barthes sont transformés pour définir la peinture comme un tissu de citations dont le sens dépend de sa destination. Le texte se termine ainsi par la phrase : "La naissance du regardeur doit se payer de la mort du peintre. »

Mêlant deux voix et s'exprimant au travers de textes préexistants, l'auteure de ce texte n'existe pas, en tous cas pas selon des considérations officielles et légales. Elle est fictionnelle. C'est d'ailleurs ainsi que l'on peut comprendre qu'un autre Statement de Levine s'appuie sur l'histoire de Pierre Ménard, le personnage d'une nouvelle de Jorge Luis Borges qui entreprend de réécrire à la lettre le Don Quichotte de Miguel de Cervantès sans le modèle pour vivre lui-même l'expérience d'écrire ce texte en son nom propre et selon son point de vue. Les Statements de Levine montrent que de mêmes mots peuvent dire d'autres vérités que celles qu'ils énoncent : une nouvelle de Moravia parle de ce qu'est une copie, un texte de Barthes sur la littérature, introduit par quelques lignes de Marc, porte sur la peinture, une fable de Borges sur la copie d'un roman permet de penser la reproduction d'images.

\section{Pénétrer les consciences}

Pendant l'année universitaire 1976-1977 Jenny Holzer suit le Whitney Independant Study Program, un cycle de formation axé sur le débat et l'analyse critique, qui accueille de jeunes artistes, curateurs et critiques d'art ayant terminé leurs études ${ }^{13}$. Les 
théories féministes et marxistes y ont une place importante et sont régulièrement débattues ${ }^{14}$. Holzer explique que sa série des Truisms (Fig. 2), commencée en 1977, est née de la confrontation avec les listes bibliographiques que leur donnait Ron Clark dans le cadre de son séminaire hebdomadaire sur les théories sociales et culturelles ${ }^{15}$.

Je voulais démêler ce que je pouvais faire ou ce que quiconque pouvait faire avec une information si dense et parfois contradictoire. Alors j'ai réécrit sa bibliothèque. Je l'ai fait comme une stratégie de développement personnel et j'ai affiché le résultat - les Truisms - dans les rues. ${ }^{16}$

Fig. 2

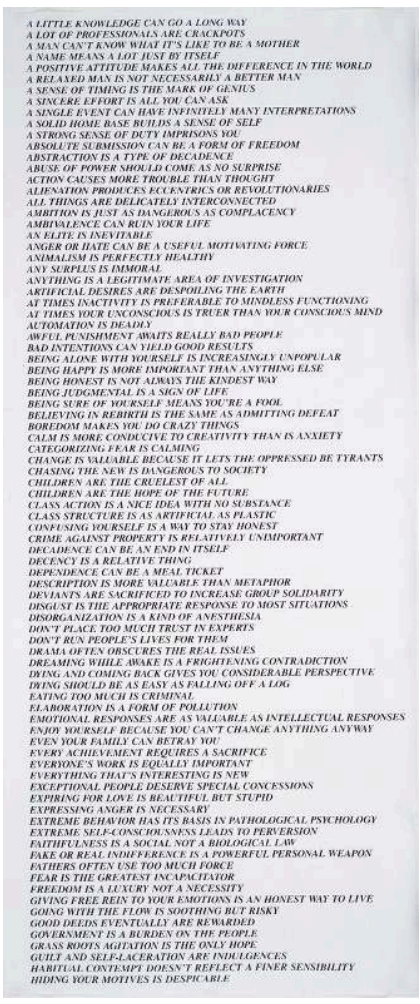

Jenny Holzer, Truisms, 1977

17 C'est donc pour rendre compte d'une multiplicité de lectures, interprétations et théories que Jenny Holzer crée ces affiches sur lesquelles sont imprimées des listes de phrases. Chacune est un conseil, une maxime ou l'explication du bien fondé d'un type de conduite. On trouve ainsi "Every achievement requires a sacrifice» [Toute réussite nécessite un sacrifice], "Ambition is just as dangerous as complacency" [L'ambition est tout aussi dangereuse que la suffisance] ou «Anger or hate can be a useful motivating force » [La colère ou la haine peuvent être d'utiles forces de motivation]. Mais la liste ne produit pas de discours cohérent. Aucun sujet ni point de vue cohérent ne s'en dégage.

18 Collés dans l'espace public parmi d'autres affiches, publicitaires, informatives ou militantes, les Truisms signalent, en s'y intégrant, que le langage est omniprésent dans ces environnements et qu'il est exploité de façon similaire pour des buts très différents. En effet la brièveté des phrases de Holzer rappelle celle des slogans qu'utilisent les politiques, les militants et les vendeurs de toutes sortes. Cette forme qui condense un discours dans une phrase s'est développée dans le but de persuader les masses ${ }^{17}$. Le slogan est court pour apparaître comme une vérité incontestable et partagée par tout le 
monde. Synthétique, il évacue tout ce qui n'est pas indispensable à son message et tout ce qui relève de la pondération. Il ne se discute pas et ne souffre aucune alternative. Enfin, son message simplificateur garantit une circulation rapide qui se fait par la répétition d'une phrase toujours identique qui ainsi ressemble à une évidence sans alternative. Parce qu'il ne relève pas de l'argumentation et qu'il se propage parmi la foule à laquelle il impose une vision, le slogan est un langage autoritaire. Pour ces raisons il a le pouvoir, ou en tout cas l'objectif, d'influencer celles et ceux qui le reçoivent. En somme sa vocation est l'incitation si ce n'est le contrôle social.

Mais les Truisms se distinguent des autres slogans qu'ils côtoient dans la rue. Ces derniers sont conçus pour promouvoir un produit ou communiquer une revendication. Ils s'expriment donc de façon implacable, directe et évidente. Les raisons de leur émission, leur provenance, ne font aucun doute. On sait clairement qui les revendiquent. Ce n'est pas le cas des Truisms dont l'auteur est inconnu et ses intentions troubles.

Jenny Holzer explique que les Truisms peuvent exprimer les idées d'une personne.

Je présentais les voix plus ou moins simultanément, et uniformément alourdies, pour suggérer que ces pensées étaient vraies pour quelqu'un. Ca semblait être une façon claire et compréhensible pour présenter un système de pensée, puisque je ne faisais pas de choix. ${ }^{18}$

21 La volonté de rendre ces phrases "vraies pour quelqu'un" révèle un penchant de l'artiste pour la création de personnages imaginaires. Mais plus encore, elle semble vouloir faire en sorte que le spectateur vive ces pensées comme si c'étaient les siennes. Holzer met ainsi le caractère persuasif du slogan au service de la création d'images et de réflexions imaginaires. Elle affirme d'ailleurs :

J'invite systématiquement le lecteur à organiser les propositions et à compléter les pensées ainsi qu'à faire résonner, amplifier ou à diminuer les sentiments que l'œuvre suscite. Je lie le langage au visuel comme une aide et aussi comme un cadeau à emporter ${ }^{19}$.

L'artiste engage une relation avec ses spectateurs dont elle excite les sentiments et les affects. Cela relève bien d'une maitrise de ce qui dans le slogan fait du langage une arme de persuasion. Mais Holzer l'exploite à des fins alternatives de celles de la propagande ou de la publicité. Il s'agit moins de convaincre que de signaler et d'exploiter ce phénomène. Les énoncés qu'elle écrit portent sur leurs effets suggestifs un regard critique mais ils le font grâce à une véritable compétence et aussi une appétence à utiliser ces possibilités d'interaction avec les sentiments humains. C'est parce qu'on ne sait de quoi elles veulent nous convaincre que ces phrases peuvent être considérées comme une approche critique du slogan. En effet, elles dévoilent un dispositif et un fonctionnement dont l'efficacité dépend de sa discrétion. Pour être influent le slogan ne peut se permettre aucune des ambiguïtés et des incohérences dont font preuve les Truisms. Pour autant ces derniers ne nous proposent pas moins de véritables expériences de suggestion. 
FEAR IS THE MOST ELEGANT WEAPON, YOUR HANDS ARE NEVER MESSY. THREATENING BODILY HARM IS CRUDE. WORK INSTEAD ON MINDS AND BELIEFS, PLAY INSECURITIES LIKE A PIANO. BE CREATIVE IN APPROACH. FORCE ANXIETY TO EXCRUCIATING LEVELS OR GENTLY UNDERMINE THE PUBLIC CONFIDENCE. PANIC DRIVES HUMAN HERDS OVER CLIFFS; AN ALTERNATIVE IS TERROR-INDUCED IMMOBILIZATION. FEAR FEEDS ON FEAR. PUT THIS EFFICIENT PROCESS IN MOTION. MANIPULATION IS NOT LIMITED TO PEOPLE. ECONOMIC, SOCIAL AND DEMOCRATIC INSTITUTIONS CAN BE SHAKEN. IT WILL BE DEMONSTRATED THAT NOTHING IS SAFE, SACRED OR SANE. THERE IS NO RESPITE FROM HORROR. ABSOLUTES ARE QUICKSILVER. RESULTS ARE SPECTACULAR.

Jenny Holzer, Inflammatory Essays, 1979-1982

C'est d'autant plus vrai dans la série suivante de Jenny Holzer. Les Inflammatory Essays (1979-1982) (Fig. 3) sont des affiches monochromes de différentes couleurs et de format carré. Chacune porte un paragraphe de quelques phrases courtes. Elles s'adressent aux lecteurs, souvent sur un ton agressif, pour exprimer ou dicter des comportements et des pensées. L'une d'entre elles commence par :

Détruis ton foutu toi avant qu'ils ne le fassent. Autrement ils te baiseront parce que tu n'es personne ${ }^{20}$.

Une autre par :

Une loi cruelle mais ancienne exige un œil pour un œil. La réponse au meurtre doit être l'exécution ${ }^{21}$.

Pour écrire ces phrases affirmatives et passionnées Holzer s'est inspirée de textes politiques. Mais comme le souligne David Joselit, contrairement aux manifestes qui véhiculent l'avis de leurs auteurs, on ne sait qui s'exprime dans les Inflammatory Essays $^{22}$. Se mêlent là les évidences incontestables du slogan et le raisonnement personnel de la déclaration. En fait ces textes associent une démonstration, dont la vocation est d'être éloquente, objective et rationnelle, à un rapport au monde affecté et émotif, sans prise de recul. En somme c'est une expérience qu'elle communique, celle de quelqu'un pensant et analysant son environnement. Ainsi Holzer exploite les ressorts du slogan pour ses capacités, ou en tout cas ses objectifs, à pénétrer la conscience humaine pour lui inoculer telle ou telle idée. Mais en véhiculant un message qui ne cache pas sa nature personnelle, l'artiste semble moins vouloir imposer une idée que proposer de faire l'expérience de la relation au monde d'un personnage imaginaire. 
En 1979 Barbara Kruger réalise une pièce qui semble partager des intentions similaires. Il s'agit d'une affiche placée dans une vitrine de la librairie new-yorkaise Printed Matter, à côté de la porte d'entrée (Fig. 4). Elle présente un texte qui parle d'un «Vous » qui semble être la personne qui la lit. L'arrivée devant le magasin est décrite ainsi que ce qui se passe à l'intérieur mais on apprend aussi ce à quoi « vous " pense. Se suivent par exemple les phrases :

Le vent sépare vos cheveux et vous êtes content.e de porter une veste. Vous n'avez pas d'a priori sur cet endroit. Vous pensez au crime et à l'argent. Vous regardez à travers la vitre. Vous montez deux marches ${ }^{23}$.

Fig. 4

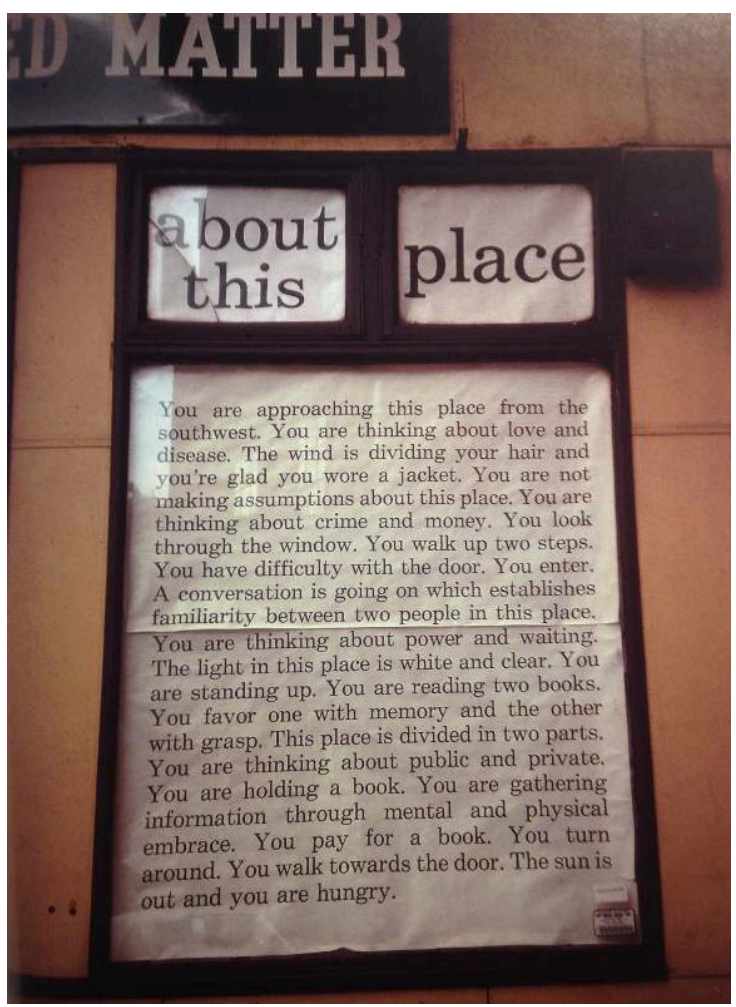

Barbara Kruger, Window Installation, 1979

Ce texte s'adresse aux clients et décrit ce qu'ils font ou ce qu'ils vont très probablement faire. Mais s'y ajoutent des sentiments inventés et des actions fictionnelles. Se mêlent la description d'une situation vécue en direct avec une fiction vue à distance, une représentation. En cela ce texte s'apparente à la voix suggestive de l'hypnotiseur, autre type de maîtrise du comportement d'un ou de plusieurs sujets. De même que les slogans de Holzer convoquent la notion de contrôle, la proposition de Kruger propose une expérience imaginaire produisant des images mentales qui imposent pensées et comportements à celles et ceux qui en font l'expérience. Les deux artistes utilisent une voix à l'auteur imprécis, indéfini. Les histoires ne sont pas racontées sur un mode personnel et singulier comme une expérience vécue et transmise ou comme un conte. $\mathrm{Au}$ contraire, elles exploitent des lieux communs.

Cela a largement été exploité ensuite par Barbara Kruger dans les affiches qu'elle réalise à partir de 1980 et qu'elle aussi commence par coller dans la rue. Elle utilise alors des images noir et blanc puisées dans des journaux, des manuels en tous genres, 
des guides de premiers soins et des magazines de sciences. Elle y pose des bandes de papier, noires ou blanches dans les premières œuvres (elles seront aussi rouges par la suite), qui portent de courtes phrases écrites avec la police de caractères Futura. Elle photographie ces collages qu'elle tire ensuite en plus grand format ${ }^{24}$. Les locuteurs et les destinataires de ces phrases, souvent énoncées par un " Nous » ou s'adressant à un "Vous", peuvent être celles et ceux qui lisent et regardent les affiches comme les personnes qu'elles représentent.

Souvent les phrases donnent des ordres ou décrivent des situations qui relèvent de la construction d'identité, de types de comportements ou de contrôles sociaux. Ainsi "Vous fleurissez sur une identité erronée " [Untitled (You Thrive on Mistaken Identity) (1981) (Fig. 5)] est inscrit sur l'image d'un visage de femme aux contours imprécis parce que se trouvant derrière une vitre opaque. "Votre regard heurte le côté de mon visage » [Untitled (Your Gaze Hits the Side of my Face) (1981)] est apposé sur une vue de profil d'une statue féminine. "Vos moments de joie ont la précision d'une stratégie militaire » [Untitled (Your Moments of Joy have the Precision of Military Strategy) (1980)] accompagne un bras masculin tenant vigoureusement une flamme olympique. « Nous avons reçu l'ordre de ne pas bouger " [Untitled (We have Received Orders not to Move) (1982)] barre l'image d'une femme, baissée et comme immobilisée par les d'aiguilles d'acuponcture qui couvrent son dos.

Fig. 5

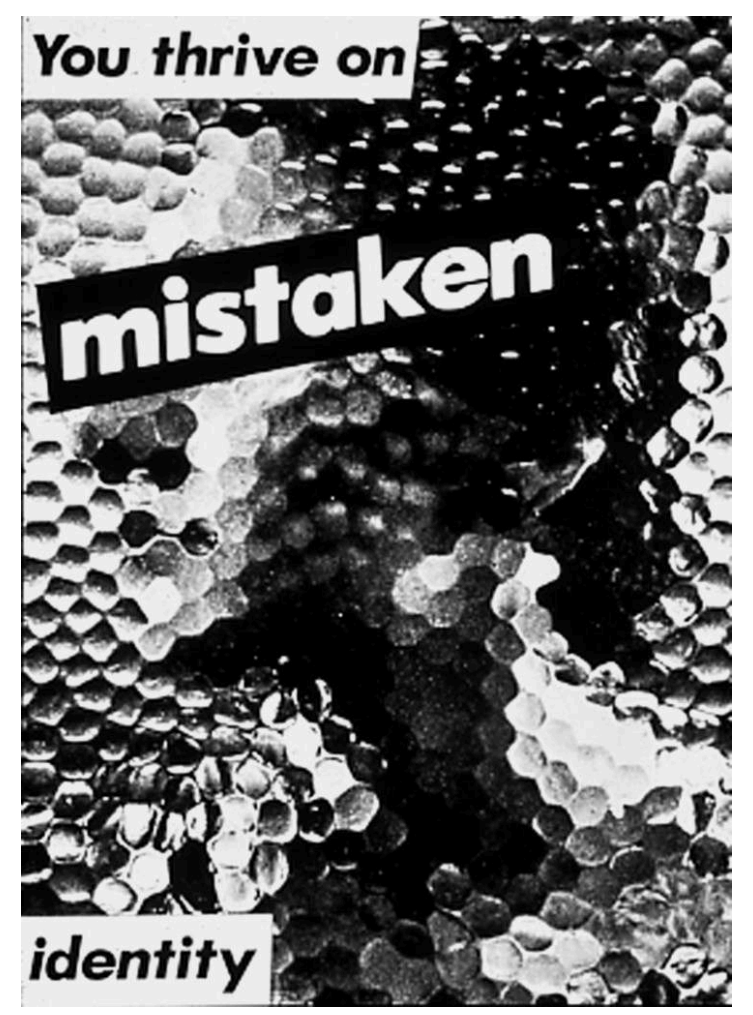

Barbara Kruger, Untitled (You Thrive on Mistaken Identity), 1981

S'il ne fait aucun doute qu'une instance s'adresse aux passants, son objectif et son message restent troubles. Contrairement aux affiches qui adressent clairement soit un message, une information à assimiler, soit une représentation idéale à désirer, la relation proposée par Kruger est ambiguë. S'ouvre ainsi un espace d'intersubjectivité 
où il est possible d'occuper simultanément la place de l'observateur et de la représentation. Ces affiches invitent à regarder des personnes et des situations depuis la distance confortable de la représentation. Mais leurs textes proposent aussi d'en être soi-même l'objet. Face à ces affiches, chacun reconnaît pouvoir être indistinctement "Nous» ou "Vous» dans les situations décrites selon qu'on s'identifie aux représentations ou qu'on en reste à distance. Comme dans le texte de Moravia convoqué par Levine on fait en même temps l'expérience de la fascination et de sa mise à distance. On fait ainsi simultanément l'expérience de chacune des places, celle de l'énonciateur et celle du récepteur mais aussi et surtout celle du désignant et celle du désigné.

31 Cette instabilité des rôles engendre une prise en considération de la façon dont le discours modèle les comportements. Qu'il s'agisse de mettre en doute le fait de posséder une identité qui soit véritablement personnelle avec Untitled (You Thrive on Mistaken Identity) ou de matérialiser la violence d'un regard dans Untitled (Your Gaze Hits the Side of my Face) c'est bien de la façon dont les femmes modèlent leur apparence comme leur identité qui est en jeu ici. Un modelage qui apparaît comme une forme de contrôle des corps, des mouvements et des actions. Toutes les attitudes, sentiments et même les joies dans Untitled (Your Moments of Joy have the Precision of Military Strategy), semblent fabriqués. Ainsi, "Kruger a transformé les mots et les images qui normalement nous dominent, soit par la force ou par une accessibilité excessive, en armes pour montrer et défaire les mécanismes de cette domination ${ }^{25}$.»

Cette domination, qui consiste en la présentation de situations idéales auxquelles se conformer, Kruger la défait pour en faire une expérience inhabituelle. Elle révèle les mécanismes de suggestion tout en les exploitant. Ses images et ses slogans utilisent des ressorts connus et habituels, ceux de la publicité comme ceux de la propagande. L'artiste a d'ailleurs eu tout le loisir de les apprendre lorsqu'elle travaillait comme graphiste pour différents magazines du groupe Condé Nast entre 1967 et $1977^{26}$. Mais l'ambiguïté de l'adresse de ses affiches les prive de but ou de message précis. Cette ambiguïté, qu'elle partage d'ailleurs avec Jenny Holzer, permet d'exploiter la force de l'outil de contrôle social qu'elles exploitent et commentent. Autrement dit, ces artistes ne renient pas l'utilisation d'un pouvoir qu'il faudrait uniquement considérer comme maléfique et dangereux. Elles en proposent une expérience alternative, similaire à celle d'un slogan imparable ou d'une image stéréotypée mais dont le but est moins de séduire ou de persuader que de faire prendre conscience de ce phénomène en en faisant l'expérience. Il s'agit véritablement d'éprouver ce qu'est la soumission à une idée, à sa pénétration dans la conscience. On a donc là affaire à une forme critique qui ne fait pas la chronique d'un phénomène mais qui le souligne en l'exploitant. En l'occurrence, il s'agit dans le travail de Kruger d'explorer la façon dont un sujet s'attache à, et est obnubilé au point de s'identifier avec, une représentation générique. Ce mécanisme consiste à présenter à des personnes singulières des idées, des phrases et des attitudes impersonnelles et idéales pour qu'elles les désirent. Dans les travaux que nous venons d'étudier est inlassablement fouillée la rencontre entre des faits réels et des fictions, des pensées singulières et des idées reçues, des individus et des personnages stéréotypés. 


\section{Écrire des images mentales}

Birth to Death List (1973) (Fig. 6) de Matt Mullican, qui est parfois aussi nommé Essex (Details of an Imaginary Life from Birth to Death), s'appuie sur l'investissement personnel dans une narration générique. Il s'agit d'une suite de 200 descriptions de la vie d'une femme. La première est «Sa naissance » [Her birth], la dernière "Sa mort » [Her death]. Entre les deux sont déroulés les sentiments qu'a pu connaître ce personnage («Les rayons du soleil lui font mal au yeux» [The sunlight hurts her eyes], « Avoir soif pendant un après-midi chaud » [Feeling thirsty one hot afternoon]), des faits marquants ou banals ("Son enfant est né » [Her baby is born], «Cuisiner des légumes » [Cooking vegetables]), des situations, des choses ou des moments qu'elle a pu voir ou vivre ( Le sel sur la table à manger " [The salt on the dining room table], «La bouche de son mari » [Her husband's mouth]).

\section{Fig. 6}

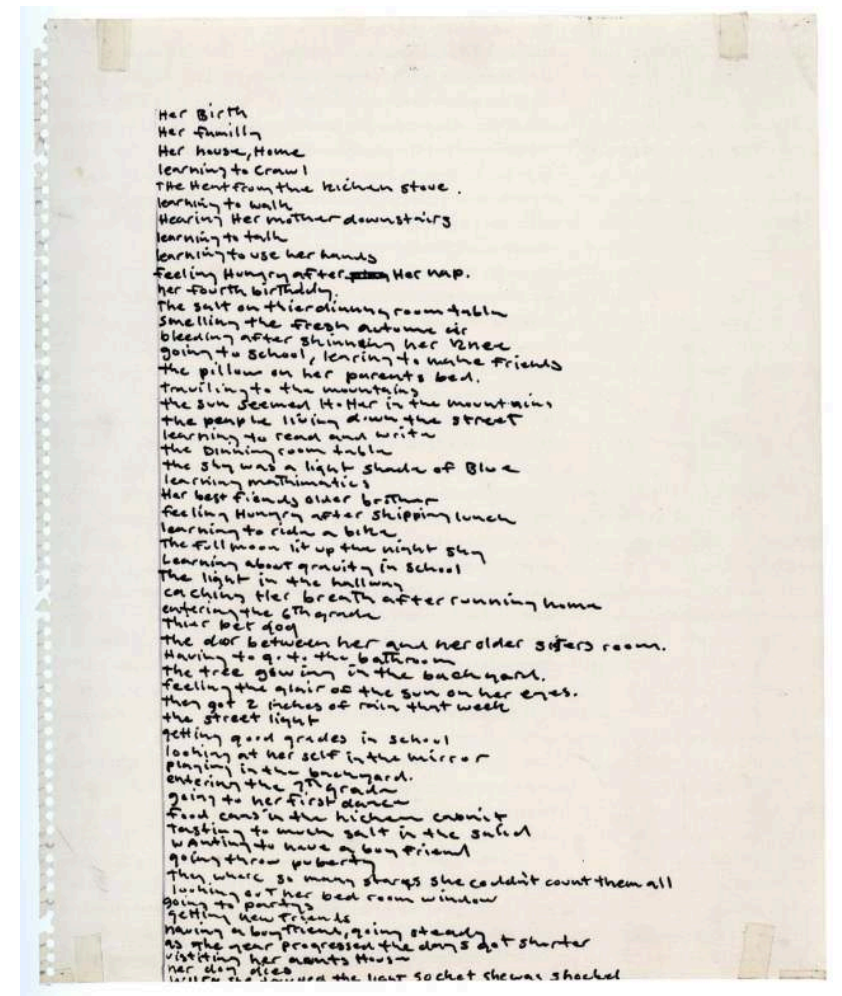

Matt Mullican, Birth to Death List, 1973

Ce texte est parfois lu par Mullican pendant des performances. Rendant compte de l'une d'elles, Allan McCollum explique que

l'effet cumulé de cette attaque sur nos capacités à former des images produit un incontestable développement de sentiments empathiques ou de nostalgie ; c'est un sentiment que vous n'auriez pas pensé expérimenter en écoutant une lecture purement répétitive de phrases très simples concernant un être humain complètement fictionnel à propos duquel on ne sait rien et dont on n'apprend rien $^{27}$.

En effet, si les situations, les actions et les personnages sont précisément décrits, ils sont aussi impersonnels. Aucun nom propre n'ancre les personnes ou les lieux dans une 
réalité donnée. Ainsi cette histoire est particulière (c'est une femme, elle se marie, fait des études d'histoire, fait l'expérience de sentiments et de situations) mais elle n'est pas biographique (on ne connaît pas son nom ni l'endroit où elle vit). Barbara Kruger. Il nous semble aussi que les deux artistes partagent un intérêt pour la façon dont une représentation générique peut être investie de façon personnelle. Pour Mullican cela passe par le détail. C'est ce sur quoi repose la fiction. L'utiliser permet de se débarrasser de la narration pour se concentrer sur les effets perceptifs. Selon Mullican on peut faire exister des mondes imaginaires en happant l'investissement mental des spectateurs. En cela il ne tente pas de narrer telle ou telle aventure mais de faire exister des mondes imaginaires par la suggestion. Ses textes racontent moins qu'ils n'affectent. C'est ce qu'il dit quand il convoque l'émotion plus que la logique. Et c'est cela qu'a vécu McCollum lorsqu'il n'a rien appris de la femme de Birth to Death List mais a ressenti de l'empathie à son sujet. L'évolution du personnage, son aventure, n'a pas d'importance dans cette œuvre qui ne propose ni morale ni position. De la narration n'est conservé que l'engagement affectif qu'elle produit. Les détails et leur banalité sont là pour capter la subjectivité de l'auditeur ou du lecteur. Suffisamment précis pour faire exister ce qu'ils décrivent, ils sont aussi assez généraux pour être investis par tous. fantastiques décrits ou représentés, c'est également ce qu'exploitent les nouvelles écrites par Richard Prince. Chez lui aussi l'identité du locuteur est instable et mouvante. Il explique :

Les pronoms que j'utilise dans les textes sont en fait des photographies. Les «il », « elle » et « ils » sont des photographies que je regarde ${ }^{29}$.

Ainsi, on peut considérer ces nouvelles comme des tentatives de rendre compte de l'expérience de regarder des images et de s'investir dans ce qu'elles représentent. Ces textes racontent le rapport entre Richard Prince et les publicités qu'il utilise aussi dans ses œuvres plastiques. En effet, durant cette période il photographie des pages de magazines qu'il cadre de façon à en exclure les slogans et les informations textuelles, produisant ainsi des scènes d'un monde fabriqué et idéal. On perçoit la façon dont il s'y investit dès les premières lignes de "The Perfect Tense » :

Beaucoup de gens aimeraient être quelqu'un d'autre. Et certains d'entre nous voudraient échanger des parties d'eux-mêmes avec d'autres gens en gardant ce qu'ils aiment et en rejetant ce qu'ils ne supportent pas ${ }^{30}$.

41 La plupart des nouvelles de Prince évoquent des personnages qui tentent de définir leur relation au monde, ce qui caractérise leur personnalité et leur apparence. Tout est très superficiel dans ces descriptions de scènes, de sensations, de pensées qui ont comme 
point commun d'évoquer un monde dans lequel tout est représentation et où les personnages ne font que reproduire des modèles qu'ils admirent. L'influence des scènes publicitaires explique aussi le caractère statique de ces histoires qui n'ont que peu de trame narrative et se présentent comme des suites de scènes. Des moments sont décrits, des pensées sont exprimées mais, outre des cas comme "Anyone Who is Anyone" qui évoque de façon décousue la quête d'un homme pour définir sa personnalité, ces textes ne narrent pas l'évolution d'un personnage face à une intrigue. Ils sont faits d'une suite de sensations, de pensées et de brèves scènes. Leur lecture s'apparente au visionnage de bandes-annonces de promotion de films qui privilégient à la narration d'une histoire l'évocation de son atmosphère.

Publiés dans des magazines ou sous la forme de livrets, les textes de Richard Prince ne sont pas inconnus du public et des critiques. Mais, alors qu'une attention est accordée à ses photographies dès la fin des années 1970, il faut attendre 1985 pour voir paraître un texte critique à leur sujet. "Mindless Pleasure : Richard Prince's Fictions " [Plaisir irréfléchi : les fictions de Richard Prince] est un essai de Brian Wallis qui accompagne la publication dans la revue Parkett de « Anyone Who Is Anyone ${ }^{31}$.

explique que, pour nous faire consommer, la publicité construit des mondes imaginaires et désirables. Prince leur donne une existence en offrant une histoire à ces personnages. Les désirs qu'avivent les publicités sont aussi le moteur des nouvelles de Prince. Wallis en conclut que le plaisir que Richard Prince nous montre est celui d'un retrait du monde qui se consomme seul depuis la distance et l'irréalité proposée par les images qu'il regarde.

Le texte de Wallis a une place importance dans l'histoire de la réception critique accordée au travail de Richard Prince. Dans le texte chronologique écrit par Lisa Phillips en 1992 pour la rétrospective de Prince au Whitney Museum, l'interprétation de Wallis est signalée comme la première à parler de plaisir à propos de cette œuvre ${ }^{32}$. Il nous semble donc important de souligner que les courtes nouvelles de l'artiste ouvrent la porte à une considération de ses photographies de publicités comme formulant une critique de ce genre d'image tout en étant, comme les originales, pourvoyeuses de plaisirs et de sentiments. Insistons également sur un point: si ce plaisir est solitaire, parce que mental, il est produit par des représentations créées en masse. On retrouve là un message aux effets hypnotiques et suggestifs qui engendre un contrôle généralisé d'une masse à qui les mêmes plaisirs sont adressés.

De façon générale on constate que les textes de tous ces artistes mobilisent les affects de leurs lecteurs. Faits d'injonctions, de descriptions de scènes ou de situations isolées, ils ne proposent pas de la narration. Par contre, ils exploitent la réception qu'en font les lecteurs pour produire des images mentales.

Lorsqu'il n'avait pas assez d'argent pour faire ses films dits "hollywoodiens", Jack Goldstein écrivait des aphorismes qui décrivent souvent des images spectaculaires et cinématographiques ${ }^{33}$. Ces phrases partagent avec les films de l'artiste une brièveté qui renforce l'évocation. «La femme aveugle ne porte pas de maquillage » [The blind woman wears no make-up], «Le héros meurt au ralenti » [The hero dies in slow motion] ou « Elle rêve de son acteur favori" [She dreams of her favourite movie star]. Ces phrases pourraient décrire des extraits de films, des scènes impressionnantes faites pour générer des émotions. En cela, elles sont aussi proches de bandes-annonces. Comme le souligne John Miller, l'un des aphorismes de Goldstein est «L'art devrait être une bande-annonce pour le futur» [Art should be a trailer for the future $]^{34}$. Une bande- 
annonce est une promesse, celle d'un film à venir. Elle est faite pour déclencher le désir pour quelque chose qui n'est pas encore là. Or il nous semble que c'est de cette façon qu'est exploité le texte par Goldstein et par les autres artistes de la Pictures Generation. Il doit diffuser un sentiment, un plaisir et il atteint plus directement cet objectif en se débarrassant d'une histoire à raconter.

Cette idée de narration sans intrigue nous paraît importante pour qualifier ce que produisent les images des artistes de la Pictures Generation. Les textes qu'ils écrivent décrivent la charge affective que véhiculent les images de la publicité et du cinéma qu'ils exploitent dans leurs œuvres plastiques. Mais ils ne présentent pas d'histoire qui en habituellement ce à quoi cette capacité d'imagination est normalement dédiée, pour ne conserver que les effets produits par les images des magazines, de la télévision et du cinéma. Leurs textes cherchent à instaurer une situation pour produire une image dans l'esprit des lecteurs et les affecter. Ces artistes soulignent ainsi ce qui compte dans leurs images: l'état mental dans lequel elle plonge leurs spectateurs, ce qu'elles produisent plus que ce qu'elles racontent.

\section{Le texte des images}

Le fait que ces artistes exploitent ce qui fascine dans les images de la publicité et du cinéma a fait l'objet d'une discussion qui accompagne leur réception critique. Douglas Crimp, à propos de ces œuvres faites d'images extraites d'un contexte pour être relues, et ainsi reconsidérées ailleurs et autrement, affirme que "sous chaque image il y a toujours une autre image $\aleph^{35}$. Cette interprétation est reprise par Craig Owens qui explique dans «L'impulsion allégorique » que l'art que lui et Crimp nomment postmoderne est allégorique dans le sens où « il y a allégorie chaque fois qu'un texte est doublé par un autre $»^{36}$.

Mais ce réemploi des formes fournies par les médias de masse a aussi été signalé comme une possible forme de participation dénuée de critique. L'idée selon laquelle en exploitant les ressorts de l'industrie du divertissement certains artistes ne feraient qu'en exploiter les effets sans les démystifier a été largement partagée et débattue ${ }^{37}$. Hal Foster y est souvent revenu. Prolongeant les interprétations de Crimp et d'Owens il affirme qu'en rester à un jeu avec des signes risque de verser dans ce qu'il nomme une "passion du signe», une seule admiration du pouvoir interprétatif, si les forces qui influent et dirigent ces fluctuations ne sont pas prises en compte ${ }^{38}$. S'accordant là avec les vues de Benjamin Buchloh, il affirme que les procédures allégoriques doivent se faire les outils d'une critique de la marchandisation ${ }^{39}$. Ce déplacement de l'analyse sémiotique vers les processus de fétichisation capitaliste est notamment développé dans un texte au titre manifeste : «Reading in Cultural Resistance » [La lecture comme résistance culturelle $]^{40}$. Foster y considère le signe comme un produit qui est consommé et désiré. Pour lui, détourner des signes ne change rien au principe puisqu'on continue d'en faire usage. Pire, exposer et manipuler des signes préexistants n'est pas une position de résistance mais celle qu'on attend d'un bon consommateur ${ }^{41}$. Ce sont, selon lui, les limites des contre-cultures et du détournement qui ne feraient que renforcer la passion du signe et resteraient au service du système qui les génère. Il propose au contraire de considérer la façon même dont le signe se construit. Pour Foster, il faut déchiffrer la façon dont s'exprime sa fonction normalisatrice. Il faut donc, littéralement, lire les images. Car, «en Occident aujourd'hui il ne peut y avoir de 
représentation simple de la réalité, de l'histoire, de la politique, de la société : on ne peut la constituer que textuellement; autrement on en réitère simplement une représentation idéologique..$^{42}$ "

50 Ainsi, toutes les représentations attirantes, factices et surtout ambivalentes sont pourvoyeuses d'un message incontrôlable car il est exprimé par le biais de sentiments. Parce qu'il est pourvoyeur de plaisirs irrationnels il doit être démasqué. Pour déjouer ce fonctionnement les critiques tels que Buchloh et Foster pensent qu'il ne faut pas manipuler les images et les sentiments qu'elles véhiculent mais les étudier précisément et traduire leurs significations - ce qu'ils nomment leurs textes - dont la lecture est la seule garante d'une interprétation stable enfin maîtrisée et rationnalisée. L'image pourvoyeuse de plaisirs est considérée avec suspicion. Le texte, entendu là comme déclaration administrative, propose un sens plus sûr.

51 La montée en puissance de ce type d'analyse à la fin des années 1970 et pendant les années 1980 accompagne une société consumériste marquée par l'élection en 1980 de Ronald Reagan. Réactionnaire et défenseur d'un désengagement de l'État dans les affaires sociales, il entend aussi déréguler l'économie. Ancien acteur et présentateur d'une émission de télévision, il est habitué à la caméra, à la production d'images et à la création de personnages. On considère qu'une partie de sa réussite est due à ses aptitudes à exploiter la télévision et la mise en scène ${ }^{43}$. Brian Massumi affirme même que Reagan avait une parfaite maîtrise de ce média qu'il utilisait pour construire un personnage médiatique inédit, exploitant le régime des affects plus qu'une position idéologique précise ${ }^{44}$.

52 Qu'il nous soit donc permis de penser que cette défiance critique vis-à-vis des images et de leurs promesses d'un plaisir au programme flou est l'effet d'un contexte politique ${ }^{45}$. Les sentiments, leviers de la fascination exploitée par médias de masses, le capitalisme consumériste et une politique libérale, sont suspects. Pour déjouer les plaisirs irrationnels qu'elles véhiculent à des fins idéologiques, les images doivent être interprétées comme des textes afin de dévoiler leurs véritables intentions. Ce refus de prendre en compte et d'analyser les sentiments nous apparaît pourtant comme un moyen de garantir une approche strictement rationnelle, entraînant avec elle une supériorité de la théorie sur l'expérience des œuvres. En tout cas, cela garantit la recherche d'un sens stable aux images que ces artistes s'approprient, le fameux texte dont ils dévoileraient la signification insidieuse.

53 Cette façon de considérer ces travaux a deux effets. D'une part elle fait des artistes des artisans de la démystification et, de l'autre, elle éradique complètement le rapport affectif que proposent les images en question. Dans cette approche s'est malheureusement perdue une véritable considération des images et de leurs effets. En ne prenant en compte les représentations qu'en termes textuels, qu'en termes de signification qu'il faudrait absolument stabiliser, on passe à côté de l'événement qu'est leur réception et des affects qu'elles produisent. Or, comme nous venons de le souligner cela est inhérent à la pratique des artistes de la Pictures Generation. Notons d'ailleurs que la vision du texte que proposent ces auteurs consiste à l'exempter de toute incompréhension et surtout à le purifier de l'utilisation qu'en font les artistes : mentir quant à leur auteur et construire des expériences ou des personnages factices.

54 Cette volonté de théoriser l'art comme un outil pour dévoiler une signification cachée s'apparente à ce que la théoricienne Eve Kosofsky Sedgwick nomme sarcastiquement l'approche paranoïaque ${ }^{46}$. Elle consiste à faire de la critique un acte de seule révélation. 
Obnubilée par le fait de dévoiler le sens stable, définissable et définitif d'une situation, l'approche paranoïaque présente une vérité qu'elle seule détient. Elle n'a qu'un objectif : ouvrir les yeux de son auditoire sur sa position de victime manipulée et ignorant son supplice. Elle s'efforce donc de lui faire prendre conscience d'une oppression qu'elle se doit d'ignorer pour garantir toujours à la critique paranoïaque son rôle de révélateur. Sedgwick souligne que le seul objectif de cette approche est la suspicion et qu'en son nom tout plaisir est proscrit parce que dangereux et outil d'asservissement. Il nous semble que c'est dans une disposition similaire que les travaux de la Pictures Generation ont été interprétés dans - et probablement à cause de - leur contexte d'apparition.

Pourtant la lecture, convoquée par Foster comme moyen de révéler le sens véritable des produits culturels, est utilisée par ces artistes pour affecter. En cela l'étude des textes qu'ils écrivent est éclairante. Ils proposent une expérience irrationnelle, produisent des images mentales, des effets d'identification et de suggestion. En cela, ces textes sont complémentaires des exploitations d'images auxquelles se livrent les artistes de la Pictures Generation. Ils accompagnent des œuvres qui ne cherchent pas à dévoiler ou à révéler le sens stable et clair et unique des images mais au contraire exploitent leurs effets. Loin de se tenir à distance de ce pouvoir dont ils démystifieraient le caractère maléfique, ils en proposent une expérience alternative. En cela ils exploitent une stratégie issue de certaines approches féministes consistant à parler avec le langage du pouvoir plutôt que de chercher à s'y confronter frontalement ${ }^{47}$. Autrement dit, le caractère critique de leurs travaux se formule dans l'exploitation effective mais aussi ambiguë de ce qui fait la force de suggestion et d'affectation des images publicitaires et cinématographiques. Ils en connaissent le pouvoir qui les fascinent, qu'ils exploitent et qu'ils apprécient à distance.

\section{NOTES}

1. D'abord publié dans le catalogue de l'exposition Pictures Douglas Crimp propose ensuite une version remaniée de ce texte dans la revue October. Voir: Douglas CRIMP, Pictures, New York, Artists Space, 1977 et Douglas CRIMP, «Pictures », October, n8, printemps 1979, p. 75-88.

2. A. D. COLEMAN, «The Directorial Mode : Notes Toward a Definition » [1976], in Vicky GoLDBERG (ed.), Photography in Print, Albuquerque, University of New Mexico Press, 1981, p. 480-491.

3. Il s'agit précisément de complexes résidentiels à Berkeley en Californie et à Deerfield Beach en Floride. Voir : Carol SQUIERS, " "Who Laughs Last" : The Photographs of Barbara Kruger », in Ann GOLDSTEIN (ed.), Thinking of You : Barbara Kruger, Museum of Contemporary Art, Los Angeles, 1999, p. 141-148.

4. Barbara KRUGER, Picture/Readings, 1978, n.p.

5. Voir par exemple : Sherrie LEVINE, "Statement ", catalogue de l'exposition Mannerism : A theory of Culture, Vancouver Art Gallery, 1982, p. 67. L'ensemble des Statements de Levine sont également publiés dans Sherrie LEVINE, "Five Comments ", Brian walus (ed.), Blasted Allegories, New York, New Museum of Contemporary Art, 1987, p. 92-93. 
6. Benjamin BUCHLOH, « De l'esthétique administrative à la critique institutionnelle » [1989], Essais historiques II, Villeurbanne, Art édition, 1992, p. 155-212.

7. Erich FRANZ, « Presence Withdrawn », Parkett, n³2, 1992, p. 95-100.

8. Craig OWENS, «Sherrie Levine at A\&M Artworks », Scott BRYSON, Barbara KRUGER, Lynne TILLMAN et Jane WEINSTOCK (ed.), op. cit., p. 114-116.

9. Voir notamment : Kate LINKER et Jane WEINSTOCK, Difference : On Representation and Sexuality, New York, The New Museum of Contemporary Art, 1984.

10. Craig OWENS, "The Discourse of Others: Feminists and Postmodernism» [1983], in Scott BRYSON, Barbara KRUGER, Lynne TILLMAN et Jane WEINSTOCK (ed.), Craig Owens : Beyond Recognition Representation, Power, and Culture, Berkeley ; Los Angeles ; Londres, University of California Press, 1992, p. 57-77.

11. Barbara KRUGER, « Statement for Documenta 7 », Saskia Bos et Rudi Fuchs (ed.), Documenta 7 , Kassel, Paul Dierichs, 1982, p. 286: «We loiter outside of trade and speech and are obliged to steal language. We are very good mimics. We replicate certain words and pictures and watch them stray from or coincide with your notions of fact and fiction ».

12. Sherrie LEVINE, "Statement", in Jo-Anne BIRNIE DANZKER (ed.), Mannerism: A Theory of Culture, Vancouvert, Vancouvert Art Gallery, 1982, p. 48.

13. Jenny Holzer, artiste née en 1950, n'est habituellement pas associée à la Pictures Generation parce que son travail avec des mots n'est pas considéré comme relevant de la manipulation d'images. Pourtant il nous semble qu'elle y a sa place sous l'angle que nous proposons ici, celui d'une image mentale.

14. Sur le Whitney Independant Study Program voir Howard SINGERMAN, «In theory and Practice : A History of the Whitney Independant Study Program », Artforum, Février 2004, p. 112-117/170-171.

15. Ron Clark, né en 1943, est l'actuel directeur du Whitney Independant Study Program qu'il a fondé en 1968. Chaque semaine dans le cadre de son séminaire un texte critique est analysé et discuté par les étudiants du programme.

16. Steven Henry MADOFF, "Steven Henry Madoff talks to Jenny Holzer », Artforum, avril 2003, p. 82: "I wanted to sort out what I was to do, or what anyone was to do, with that much dense and sometimes contradictory information. So I rewrote his library. I did it as a self-help maneuver, and posted the result - the Truisms - in the streets ".

17. Olivier ReBoul, «Slogan et poésie », in Anne BALDESSARI (ed.), Art \& Publicité, Paris, Centre George Pompidou, 1991, p. 88-97.

18. Steven Henry MADOFF, "Steven Henry Madoff talks to Jenny Holzer », Artforum, avril 2003, p. 82: «I presented the voices more or less simultaneously, and weighted evenly, to suggest that the thoughts were true to somebody. It seemed like a comprehensive and clean way to present belief systems, since I was not choosing ".

19. Benjamin H.D. BUCHLOH, "An Interview with Jenny Holzer ", in David BRESLIN (ed.), Jenny Holzer : Protect Protect, Riehen/Bâle ; Chicago, Fondation Beyeler; Museum of Contemporary Art, 2008, p. 119 : «I routinely invite the reader to sort through the offerings and complete the thoughts, and to echo, amplify, or shrink from the feelings the work elicits. I tie the language to the visual as an assist, and as a take-away gift ».

20. "Ruin your fucking self before they do. Otherwise they'll screw you because you're a nobody ".

21. "A cruel but ancient law demands an eye for an eye. Murder must be answered by execution".

22. David Joselit, «Voices, Bodies ans Spaces: The Art of Jenny Holzer », in David JoseliT, Joan SIMON et Renata SALECL (ed.), Jenny Holzer, Londres, Phaidon, 1998, p. 42-77.

23. "The wind is dividing your hair and you're glad you wore a jacket. You are not making assumptions about this place. You are thinking about crime and money. You look through the window. You walk up two steps ». 
24. Alexander ALBERRO, "Picturing Relations: Images, Text, and Social Engagement", in Alexander ALBERRo, Martha GEVER, Miwon KWON et Carol SQUIERS, Barbara Kruger, New York, Rizzoli, 2010, p. 193-200.

25. Miwon Kwon, A message from Barbara Kruger: Empathy Can Change the World, in Alexander ALBERRO, Martha GEVER, Miwon KwON et Carol SQUIERS, op. cit., p. 94 : «Kruger turned words and pictures that normally dominate us, either through force or excessive accessibility, into weapons to expose and undo the mechanisms of that domination ".

26. Pour une histoire détaillée de la carrière de graphiste de Barbara Kruger voir Carol SQUIERS, "Barbara Kruger at Mademoiselle ", in Alexander ALBERRO, Martha GEVER, Miwon KWON et Carol SQUIERS, Barbara Kruger, New York, Rizzoli, 2010, p. 225-232.

27. Allan mccollum, « Matt Mullican's World » [1980], in Miriam KATZEFF, Thomas LAwson et Susan MORGAN (ed.), Real Life Magazine: Selected Writing and Projects 1979-1994, New York, Primary Information, 2007, p. 81 : «The accumulated effect of this assault on our image-forming capacity is an unquestionable growth of empathic feeling, or nostalgia; it is a feeling we would not have anticipated experiencing as a result of listening to a purely rote reading of such simple phrases which refer to a completely fictional human being of whom we know nothing, and have learned nothing ".

28. Michael TARANTINO «Details: An Interview with Matt Mullican », in Michael TARANTINo (ed.), Matt Mullican: More Details from an Imaginary Universe, Turin, Hopefulmonster, 2000, p. 11 : "The very word "detail" indicates a context beyond itself-so a detail of a fiction doesn't just "go". It's like a Catch-22. It doesn't work in terms of logic, but emotionally, it works very well because we're constantly doing this. Our understanding of the world is through our imagination. I can imagine what's happening in that house across the street. "

29. Peter halley, «Richard Prince interviewed by Peter Halley », ZG, n¹0, printemps 1984, p. 6. Traduit dans : Vincent PÉCOIL, «Préface », in Richard PRINCE, Pourquoi je vais au cinéma seul, Dijon, Les presses du réel, 2013, p. 7.

30. Traduction dans op. cit., p. 18.

31. Brian WALLIS, « Mindless Pleasure : Richard Prince's Fictions ", Parkett, ${ }^{\circ} 6$, Septembre 1985, p. 61-62.

32. Lisa PHILLIPS, «People Keep Asking : An introduction », in Lisa PHILlIPS (ed.), Richard Prince, New York, Whitney Museum of American Art, 1992, p. 21-53.

33. Le terme d'« hollywoodiens » est utilisé par Morgan Fisher pour désigner les films très courts réalisés par Jack Goldstein de 1974 à 1976, pour la réalisation desquels il utilise les moyens et les techniques qu'il peut se permettre de louer dans les studios d'Hollywood. Morgan FISHER, « Discussion avec Jack Goldstein » [1977], in Yves AUPETITALLOT et Lionel BOVIER (ed.), op. cit., p.16

34. John MILLER, «A trailer for the future », in Daniel BUCHHOLZ et Christopher müLleR (ed.), Jack Goldstein, Films, Records, Performances and Aphorisms 1971 - 1984, Cologne, Verlag der Buchhandlung Walther König, 2003, p. 6-13.

35. Douglas CRIMP, art. cit., p. 87.

36. Craig OWENS, «L'impulsion allégorique: vers une théorie du postmodernisme », in Charles HARRISON et Paul WOoD (ed.), Art en théorie, 1900-1990, Paris, Hazan, 1997, p. 1147.

37. À la fin des années 1970 et au début des années 1980 les textes sur ces questions sont nombreux, citons : Benjamin BUCHLOH, «Allegorical Procedures: Appropriation and Montage in Contemporary Art», Artforum, septembre 1982, p. 43-56; Douglas CRIMP, "Appropriating Appropriation » [1982], On the Museum's Ruins, Cambridge et Londres, Mit Press, 1993, p. 126-137; Martha ROSLER, «Notes on Quotes » [1982], in IDEM, Decoys and Disruptions: Selected Writings, 1975-2001, Cambridge et Londres, MIT Press, 2004 ; Abigail soLOMON-GODEAU, « Playing in the field of images ", Afterimage, $\mathrm{n}^{\circ} 1$ et 2, été 1982, p. 10-13.

38. Hal FOSTER, « La passion du signe », Le retour du réel, Bruxelles, La lettre volée, 2005, p. 99-125. 
39. Benjamin BUCHLOH, "Allégorie et appropriation dans l'art contemporain » [1982], Essais historiques II, Villeurbanne, Art édition, 1992, p. 107-153.

40. Hal FOSTER, « Reading in Cultural Resistance », op. cit., p. 157-179.

41. Hal FOSTER, art. cit., p. 171.

42. Hal Foster, « For a Concept of the Political in Contemporary Art » [1984], in op. cit., p. 155 : « In the west today there can be no simple representation of reality, history, politics, society: they can only be constituted textually; otherwise one merely reiterates ideological representation of them. "

43. André KASPI, Les Américains - 2 : Les États-Unis de 1945 à nos jours, Paris, Editions du Seuil, 2014.

44. Brian MASsumi, Parables for the Virtual: Movement, Affect, Sensation, Durham et Londres, Duke University Press, 2002.

45. Howard SINGERMAN, "Pictures and position in the $80^{\prime}$ ", in Amelia JONES (ed.), A Companion to Contemporary Art Since 1945, Malden ; Oxford ; Carlton, Blackwell Publishing, 2006, p. 83-106.

46. Eve KOSOFSKY SEDGWICK, « Paranoid reading and reparative reading, or, you're so paranoid, you probably think this essay is about you ", Touching Feeling: Affect, Pedagogy, Performativity, Durham et Londres, Duke University Press, 2003, p. 123-151.

47. Nous pensons notamment à la notion de mascarade développée par Joan Rivière et celle de pose chez Craig Owens. Joan RIVIERE, "Womanliness as Masquerade", in Victor BURGIN, James DONALD, Cora KAPLAN (ed.), Formations of Fantasy, Methuen, Londres, 1986, p. 35-44. Craig owens, " Posing », Beyond Recognition - Representation, Power, and Culture, Berkeley ; Los Angeles; Londres, University of California Press, 1992, p. 201-217.

\section{RÉSUMÉS}

A la fin des années 1970 et au début des années 1980 aux Etats-Unis, apparaissent des œuvres faites d'images empruntées aux médias de masse. A titre d'exemple : Barbara Kruger réalise des affiches qui ressemblent à celles de campagne publicitaires. Richard Prince photographie des images publicitaires. Les images que ces deux artistes, et de nombreux autres, utilisent sont originellement faites pour avoir sur leur public un pouvoir de suggestion: leur faire vivre des expériences par procuration, leur donner envie de consommer tel ou tel produit. Cette manipulation psychologique est centrale dans les textes qu'écrivent ces mêmes artistes. Jack Goldstein, Jenny Holzer, Barbara Kruger, Louise Lawler, Sherrie Levine, Matt Mullican et Richard Prince proposent, en marge de leurs œuvres plastiques, des textes qui produisent des images ou des sensations chez leurs lecteurs. La façon dont ces artistes écrivent de tels effets pourrait être un bon indicateur de la relation qu'ils tentent d'établir avec leur public lorsqu'ils manipulent le pouvoir de suggestion des représentations.

During the late 70'S and early 80's in the USA, artworks borrow images from the mass media. For instance: Barbara Kruger makes posters looking like publicity campaigns. Richard Prince photographs advertisement. Images that these two artists and many others use are originally made to have a power of suggestion on their public: they make people live by proxy, they give them will to buy products. This psychological manipulation is also central in the texts of these artists. Jack Goldstein, Jenny Holzer, Barbara Kruger, Louise Lawler, Sherrie Levine, Matt Mullican and Richard Prince propose, at the margins of their artistic practice, texts that produce images or sensations on their readers. The way those artists write these effects could be a good 
indicator of the relation they are trying to establish on their public when they manipulate the suggestive power of representations.

INDEX

Index chronologique : XXe siècle

Keywords : contemporary art, Pictures Generation, advertising, cinema, suggestion

Mots-clés : art contemporain, Pictures Generation, publicité, cinéma, suggestion

Thèmes : art contemporain

Index géographique : Etats-Unis

\section{AUTEUR}

\section{FRANÇOIS AUBART}

François Aubart est critique d'art et commissaire d'expositions indépendant. Il enseigne l'histoire et la théorie de l'art à l'Ensba Lyon. Il est co-fondateur de la revue $\Delta$ 人 et du projet éditorial future [http://f-u-t-u-r-e.org/]. Il prépare un doctorat sur la Pictures Generation. 